\title{
Therapeutic Approach to Acute Hepatitis C
}

\author{
Rodrigo Nogueira Angerami ${ }^{1}$ and Fernando Lopes Gonçales Júnior ${ }^{2}$ \\ ${ }^{l}$ Epidemiological Surveillance Unit, Hospital Epidemiology Service, Hospital das Clínicas, State University at Campinas and Campinas \\ Referral Center for STD/AIDS; ${ }^{2}$ Study Group on Hepatitis, Infectious Diseases Division, Clinical Medicine Department, School of Medical \\ Sciences, State University at Campinas; Campinas, Brazil
}

It is estimated that 150 to 200 million individuals are currently infected with the hepatitis $\mathrm{C}$ virus (HCV) [1-3], and that, annually, there are 3 to 4 million new cases of infection worldwide [4]. In view of the impossibility of immunoprevention [5] - either through vaccines or the use of post-exposure immunoglobulin - and the risk of chronicity in individuals exposed to the disease, which is estimated to be between $50 \%$ and $85 \%$ [2,4,6-9,33], the definition of strategies aimed at early detection and treatment of infected individuals, preferably during the acute phase of the infection $[2,4]$, has been widely discussed in recent years.

In individuals presenting symptoms consistent with acute hepatitis $\mathrm{C}$ (AHC), it is known that the possibility of spontaneous viral clearance, among various factors, depends especially on a potent initial cellular immune response mediated by cytotoxic T lymphocytes - initially by $\mathrm{HCV}$-specific CD8+ T lymphocytes and, subsequently, CD4+ T lymphocytes [1012]. Other factors associated with a higher rate of sustained virological response (SVR) are the specific characteristics of the patients. Individuals who are Asian or Caucasian, female, young, and HIV-negative, as well as presenting a rapid drop in HCV RNA levels and presenting specific human leukocyte antigen class II alleles, together with the previously mentioned $\mathrm{T}$ cytotoxic response, also respond better to treatment $[16,17,33]$. Better responses are also found in cases of infections with shorter incubation period produced by smaller inoculates. In addition, in a prospective study conducted by Santantoni et al., a higher rate of spontaneous viral clearance was observed in patients infected with genotype 3 [7].

A diagnosis of acute infection with $\mathrm{HCV}$ remains a rare event in clinical practice. In 70\%-80\% of infected patients, AHC is asymptomatic, and $75 \%$ are anicteric $[4,8,13-15,33]$. Individuals with $\mathrm{AHC}$ present elevated serum levels of alanine aminotransferase (ALT) and aspartate aminotransferase (AST), with or without a clinical profile of acute hepatitis, detection of (previously undetectable) HCV RNA and seroconversion for anti-HCV antibodies. It is estimated that infection with $\mathrm{HCV}$ accounts for only $20 \%$ of all diagnosed cases of acute hepatitis [13]. In the United States, there are 40,000 cases of AHC annually [12], and only a small percentage of those are clinically diagnosed in this phase.

Considering the high risk of developing chronic hepatitis $\mathrm{C}$ and the favorable evidence, in terms of therapeutic response, it is fundamental to improve our capacity to properly detect and treat cases of acute infection with HCV [12].

\section{At-Risk Populations}

Currently, it is thought that, in $90 \%$ of cases of infection with $\mathrm{HCV}$, it is possible to identify the associated risk factors [14].

In the last two decades, especially after serologic triage was adopted as obligatory for blood donors, and due to the increased accuracy of serologic tests, there has been a drastic reduction in the number of new infections with HCV through blood and blood products. This resulted in significant changes in the epidemiological pattern of the disease $[2,16,18]$. However, new infections still occur via parenteral route, and, less frequently, by sexual or vertical transmission. Currently, the principal groups at risk for infection with $\mathrm{HCV}$ are as follows: health professionals exposed through cut or puncture accidents involving sharp objects contaminated with infectious material; intravenous drug users; individuals engaging in sex with HCV-positive partners; patients on hemodialysis; and patients submitted to various invasive procedures [4,12,14,18-21].

In the United States, intravenous drug users account for $68 \%$ of the new cases of HCV infection, compared with $18 \%$ for individuals engaging in sex with $\mathrm{HCV}$-positive partners, $4 \%$ for health professionals exposed through cut or puncture accidents, $1 \%$ for individuals infected through other routes, and $9 \%$ for those in whom the source of infection cannot be identified [22].

Currently, it is believed that the risk of vertical transmission is significantly potentialized in cases of $\mathrm{HIV} / \mathrm{HCV}$ co-infection, in which the rate of such transmission can be as high as $17 \%$ [37].

Special attention has been given to the occupational risk of infection with $\mathrm{HCV}$ in health professionals, and the estimated risk of cut or puncture accidents with sharp objects contaminated with infectious materials ranges from $1 \%$ to $2 \%$ [5,22].

Although risks, impacts and recommendations are still controversial, it is estimated that the risk of sexual transmission ranges from $0 \%$ to $3 \%[34,37]$, increasing especially in specific situations, such as co-infection with HIV or other sexually transmitted diseases, and between homosexual partners.

It is also known that the risk of HCV infection is higher in individuals with chronic kidney disease under treatment with hemodialysis. It is believed that, among such individuals treated in the United States, the prevalence of anti-HCV reactivity ranges from $15 \%$ to $50 \%$ [37].

* This article is part of the Proceedings of the Consensus of the Brazilian Society of Infectious Diseases on the Management and Treatment of Hepatitis C presented in the supplement of the Brazilian Journal of Infectious Diseases. 
The definition of protocols for clinical and laboratory periodic follow-up evaluation in these segments at increased risk is fundamental in order to improve the detection of AHC.

\section{Laboratory Diagnosis}

The laboratory techniques most commonly used in the detection of anti-HCV antibodies are fundamentally based on third- and fourth-generation enzyme-linked immunosorbent assays. Such serologic tests have demonstrated great specificity (>99\%) and sensitivity (95\%-99\%) [38]. However, one of the possible limitations in the diagnosis of acute infection with $\mathrm{HCV}$ results from the possibility of late seroconversion, occurring at 4 to 10 weeks after exposure [23]. Regarding detection of anti-HCV antibodies, we observe $50 \%$ to $70 \%$ positivity at symptom onset; in addition, from postinfection month 3 onward, antibodies are detected in $90 \%$ of the cases [38].

However, HCV RNA determination constitutes an important tool for early detection of acute infections, since $\mathrm{HCV}$ is detectable by 1 to 3 weeks after transmission $[23,38]$. Immediately after HCV RNA detection and concomitantly with the appearance of possible signs and symptoms - 2 to 8 weeks after infection - it is possible to observe increased ALT levels [38]. Detection of HCV RNA, in the absence of anti-HCV antibodies, strongly suggests acute infection, especially when subsequent anti-HCV seroconversion is observed. The use of transcription-mediated amplification (TMA), together with other available techniques, constitutes an additional strategy for early detection of acute HCV infection [23].

\section{Therapeutic Approaches}

The high rate of chronicity observed after infection with $\mathrm{HCV}$ justifies the increasing interest in possible therapeutic approaches in cases of acute infection.

Various approaches have been proposed in literature, all of which aim at preventing the progression to chronic disease. Nevertheless, to date, the ideal treatment regimen to be adopted in clinical practice has not been established $[4,6,8,9,14,18,19,24]$. Comparing results from different clinical trials is difficult for the following reasons: acute hepatitis $\mathrm{C}$ is frequently asymptomatic; different criteria are used in case definition; samples of patients are small and heterogeneous; there are no control groups; different types and doses of interferon (IFN) are used; there are different end points, and follow-up time varies enormously. Therefore, it is impossible to define an ideal therapeutic regimen.

When we evaluate the data available in literature, we observe excellent results in patients treated in the acute phase of the infection, even under monotherapy $[15,16]$. The principal post-treatment outcome measure is the rate at which patients achieve an SVR, defined as the absence of HCV RNA detected in serum by qualitative polymerase chain reaction (PCR) at 24 weeks after the end of the treatment $[25,26]$. Among patients treated in the acute phase of the infection, this rate ranges from $37 \%$ to $98 \%$ [2,4,8,18,20,24], which is higher than the 54-
$56 \%$ observed among patients with chronic hepatitis C, even among those under combined therapy with pegylated IFN (PEG-IFN) and ribavirin (RBV) [25,26].

Various trials evaluate the efficacy of different regimens using conventional IFN alpha (IFN $\alpha$ ) in the treatment of AHC. The best results to date were those reported by Jaeckel et al., whose treatment regimen consisted of monotherapy with IFN $\alpha$-2b with inducement - 5 million IU/day, s.c., for 4 weeks, followed by 5 million IU, 3 times a week, for 20 weeks [2]. In that study, the use of IFN, even without RBV, made it possible to obtain high SVR levels. However, the Jaeckel et al. study raises the following considerations: $68 \%$ of the patients included in the study were icteric and therefore presented a higher probability of having self-limited infection, which could, in part, explain the high SVR rate reported. As previously mentioned, symptomatic individuals can evolve to spontaneous viral clearance. In addition, Kamal et al. observed a higher SVR rate in treated symptomatic individuals when compared to those asymptomatic individuals treated with the same therapeutic regimen (96\% and $76 \%$, respectively) [8]. However, Santantonio et al. reported an SVR rate of $94 \%$ among individuals who did not present spontaneous viral clearance by week 12 after acute symptom onset, when treated with PEG-IFN $\alpha$-2b, in monotherapy, for 24 weeks [12]. Such finding corroborates other reports demonstrating that it is safe to adopt the expected conduct up to week 21 in order to await possible spontaneous viral clearance [15,16,20,28,36].

In view of these facts, it should be noted that, among individuals acutely infected with $\mathrm{HCV}$, those who are asymptomatic, especially those who are anicteric and present normal ALT levels, are more likely to present spontaneous viral clearance $[8,12,14,18,20]$ - typically between weeks 5 and 12 [27,28]. This viral clearance can occur, in rare situations (in $10-50 \%$ of cases), up to 24 weeks after symptom onset $[8,9,12-$ $15,17,18,21]$. Various authors suggest that the treatment be initiated, at most, 12 to 16 weeks after symptom onset, avoiding unnecessary exposure to IFN in patients who can clear HCV spontaneously $[4,10,12,18,33]$.

However, some authors suggest that, in cases of asymptomatic acute infection, treatment should be introduced at the time of diagnosis [12].

It should be noted, however, that lower SVR rates have been observed in cases of late treatment. These rates were initially described by Nomura et al. [18] who, using IFN $\alpha$ (6 million IU, i.m., daily, for four weeks), reported an SVR in $87 \%$ of early treated individuals - 8 weeks after detection of symptoms - and in $40 \%$ of patients treated after AHC symptom onset. This study demonstrated that early introduction of treatment enabled a high SVR rate, even in short-term treatment regimen. More recently, in a study conducted by Kamal et al., the SVR rate in individuals treated after week 20 was $76.6 \%$, whereas, in individuals who started therapy at weeks 8 and 10, the rates were, respectively, $95.3 \%$ and $93.2 \%$ [20].

Similarly to what was observed in the treatment of chronic hepatitis C [14,29,30], negative HCV RNA at treatment week 1 
was associated with a greater probability of achieving an SVR [31]. In the study carried out by Kamal et al., achieving a rapid virological response (RVR), defined as HCV RNA negativity or a $\geq 2 \log _{10}$ drop in HCV RNA, by treatment week 4, was found to have positive and negative predictive values of $88 \%$ and $98 \%$, respectively, for achieving an SVR. In another study, involving individuals with AHC treated with IFN as monotherapy in daily doses for 4 weeks, HCV RNA negativity in the first week of treatment was found to have a positive predictive value of $87 \%$ for achieving an SVR [18].

The combination of PEG-IFN $\alpha$ and RBV is currently considered the first-line treatment regimen for patients with chronic hepatitis $\mathrm{C}$, those infected with genotype 2 or 3 and treated thusly achieving an SVR at a rate of $82 \%$ [20]. However, the efficacy, safety, duration and appropriate timing of PEGIFN treatment in cases of AHC have not yet been well established.

A multicenter, prospective, randomized controlled study, using PEG-IFN $\alpha$-2b and involving a 48-week post-treatment follow-up period, reported SVR rates of $95.3 \%$ and $93.2 \%$, respectively, in groups of patients in which treatment was initiated at week 8 or week 12 after symptom onset [20]. Studies that compared treatment results obtained with conventional IFN to those obtained with PEG-IFN found similarly high efficacy in both formulations [4]. Kamal et al., comparing the efficacy of PEG-IFN $\alpha$, with and without RBV, reported $85 \%$ and $80 \%$ SVR rates, respectively [20]. It should be highlighted, however, that no evidence justifies the routine use of the combination with RBV up to the present moment.

In a multicenter study, patients with acute hepatitis $\mathrm{C}$ received PEG-IFN $\alpha-2 b$ (in a dose of 1.0-1.5 $\mu \mathrm{g} / \mathrm{kg} /$ week) initiated at the time of diagnosis and continued for 12 weeks; HCV RNA negativity was found in $87 \%$ at treatment week 4, in $91 \%$ at the end of the treatment (week 12), and in $73 \%$ at 24 weeks after the end of the treatment [9]. In patients treated with higher doses of PEG-IFN ( $\geq 1.2 \mu \mathrm{g} / \mathrm{kg} /$ week for 12 weeks), the same authors reported an $84 \%$ SVR rate, comparable to that found for 24-week regimens [9]. In a prospective, uncontrolled study, De Rosa et al., using PEG-IFN $\alpha$-2b (1.0$1.6 \mu \mathrm{g} / \mathrm{kg} /$ week, during 12 weeks) initiated immediately after diagnosis, reported an overall rate of $74 \%$, although higher rates were reported when higher doses of PEG-IFN were used (82\% when $\geq 1.33 \mu \mathrm{g} / \mathrm{kg} /$ week doses were used) [21].

Similar to what was observed in the treatment for chronic hepatitis $\mathrm{C}$, compliance to treatment regimen has proven an important SVR predictive factor in the treatment of AHC. This was demonstrated in a multicenter study, in which a $71 \%$ overall response rate was found, compared with an $89 \%$ rate found in the population with expected compliance to treatment [32].

When SVR rates were evaluated in difficult-to-treat populations, two studies involving intravenous drug users reported SVR rates between $72 \%$ and $74 \%$ [9.21]. In both situations, regular follow-up and multidisciplinary approach were used in order to provide adequate compliance to the proposed treatment regimen.
Kamal et al. reported that HCV genotype is also an element to be considered as an SVR predictive factor in patients with AHC. Therefore, similar to what was reported in cases of chronic hepatitis $\mathrm{C}$, there were associations among genotypes, treatment time, and SVR rates, in patients with acute hepatitis C. Distinct SVR rates were found, according to the genotype involved: $63.8 \%$ for genotype 1 ; and $100 \%$ for genotypes 2 and 3 . However, when a prolonged (24-week) treatment regimen was adopted, an SVR rate of $88 \%$ was observed in patients infected with genotype 1 , higher than the $38 \%$ and $60 \%$ reported for 8-week and 12-week regimens, respectively [8].

In addition to genotypes other than genotype 1 , relevant positive predictive factors for achieving an SVR include lower viral load at the start of treatment, early initiation of treatment, and rapid negative HCV RNA after the initiation of treatment $[8,18]$. Calleri et al. reported that, in addition to achieving an RVR (by week 4 of treatment) and low pre-treatment viremia, PEG-IFN doses $\geq 1.2 \mu \mathrm{g} / \mathrm{kg} /$ week were positive predictive factors for achieving an SVR [9].

It is not currently possible to determine the best treatment regimen for AHC cases. Nor can we establish a minimum followup time to be adopted after the end of the treatment. Wiegand et al., in a prospective study using biochemical, ultrasound, and virological monitoring (HCV-PCR, TMA and RNA detection in peripheral mononuclear cells) of patients treated with IFN á, in monotherapy, did not report any evidence of viral recurrence or hepatocytic lesion during the period studied (mean, 135 weeks; range, 52-224 weeks) [10]. However, as suggested by Alberti et al. [16] in a meta-analysis and subsequently by other authors, the minimum clinical, biochemical, and virological follow-up time should not be less than 48 weeks.

\section{Conclusions}

High SVR rates have been reported in patients treated for acute hepatitis $C$. In view of this, various national and international consensuses have recommended the treatment of this infection $[6,14,19,34,35]$. However, there is no consensus regarding the proper timing of the initiation of treatment onset duration of treatment. The best treatment regimen to be used, as well as the length of the post-treatment follow-up period, is also debatable.

Taking into account the fact that acute hepatitis $\mathrm{C}$ is generally asymptomatic, serologic triage and often HCV RNA testing are universally recommended strategies for correct diagnostic approach of intravenous drug users, patients infected with HIV, patients on hemodialysis, children of mothers with $\mathrm{HCV}$, sexual partners of $\mathrm{HCV}$-positive individuals, and health professionals having been exposed to HCV. These segments are currently considered the principal at-risk groups for acute infection with $\mathrm{HCV}$ and, therefore, potentially considered for treatment.

Diagnostic laboratory tests should include anti-HCV antibody tests (detectable between post-infection weeks 4 and 10), determination of serum levels of aminotransferases 
such as ALT (high between post-exposure weeks 2 and 5) and especially detection of HCV RNA (detectable by 1 week after infection). The performance of liver biopsy for the diagnosis of acute hepatitis $C$ is restricted to situations in which the clinical profile is consistent, although anti-HCV seroconversion or recent HCV RNA detection are not characteristic.

Individuals with acute hepatitis $\mathrm{C}$ are those who present increased ALT, accompanied or not by clinical profile consistent with acute hepatitis, with detection of (previously undetectable) HCV RNA and seroconversion for anti-HCV.

Taking into account the possibility of spontaneous viral clearance of $\mathrm{HCV}$, especially reported in symptomatic individuals with acute infection, it is recommended that, in symptomatic individuals, the treatment be initiated from week 12 after symptom onset onward.

In the case of asymptomatic patients with acute infection, treatment should be initiated at the time of diagnosis, since there is less probability of spontaneous viral clearance and due to the fact that late specific therapy is associated with a lower SVR rate.

Both IFN formulations - conventional IFN and PEG-IFN are considered efficacious options for the treatment of acute hepatitis C. However, there has been an increasing tendency toward using PEG-IFN as a first option, especially in individuals infected with genotype 1 . The option of high doses of PEG-IFN should be especially considered in situations in which short-course treatment regimens are used or in cases of infection with genotype 1 . When there is an option for using the conventional IFN treatment regimen, adopting a daily induction dose, followed by reduction of the number of doses, up to the end of the treatment, seems to be the most efficacious option. There is as yet no conclusive evidence that the combination of IFN and RBV is superior to monotherapy with IFN.

Therefore, as observed in studies of chronic hepatitis C with $\mathrm{HCV}$ genotype 1, prolonged treatment regimens are recommended in cases of acute hepatitis $\mathrm{C}$ with $\mathrm{HCV}$ genotype 1 , although 24-week treatments can also be considered. In acute infections with genotype 2 or 3 , short-course treatments (e.g., 12-week courses) can be feasible options.

Prolonged (24-week) treatment or combined therapy with RBV can be considered for patients who do not present an early virological response in the first 4 weeks of treatment.

Monotherapy regimens, with shorter duration and supervised administration of IFN, especially in groups of difficult-to-treat patients, would be desirable.

The ideal duration of post-treatment evaluation - clinical, biochemical (ALT levels) and virological (detection of HCV RNA) - has not yet been well established. However, durations of at least 48 weeks should be considered.

\section{References}

1. Alter M.J., Kruszon-Moran D., Nainan O.V., et al. The prevalence of hepatitis C virus infection in the United States, 1988 through 1994. New England Journal of Medicine 1999:341:556-62.
2. Jaeckel E., Cornberg M., Wedemeyer H., et al. Treatment of acute hepatitis $\mathrm{C}$ with interferon alfa-2b. New England Journal of Medicine 2001;345:1452-7.

3. Kamal S.M., Ismail A., Graham C.S., et al. Pegylated interferon alpha therapy in acute hepatitis $\mathrm{C}$ : relation to hepatitis $\mathrm{C}$ virusspecific T cell response kinetics. Hepatology 2004;39:172131 .

4. Weigrand K., Stremmel W., Encke J. Treatment of hepatitis C virus infection. World Journal of Gastroenterology 2007;13(13):1897-1905.

5. Updated US Public Health Service guideline for the management of occupational exposures to $\mathrm{HBV}, \mathrm{HCV}$ and $\mathrm{HIV}$ and recommendations for postexposure prophylaxis. MMWR 2001:50:RR1.

6. EASL International Consensus Conference on Hepatitis C, Consensus Statement, 1999. Journal of Hepatology 1999;30:956-61.

7. Santantonio T., Fasano M., Sinisi E., et al. Efficacy of a 24-week course of peg-interferon $a-2 b$ monotherapy in patients with acute hepatitis $\mathrm{C}$ after failure of spontaneous clearance. Journal of Hepatology 2005;42:329-33.

8. Kamal S.M., Moustafa K.N., Chen J., et al. Duration of peginterferon therapy in acute hepatitis $\mathrm{C}$ : a randomized trial. Hepatology 2006;43:923-31.

9. Calleri G., Cariti G., Gaiottino F., et al. A short course of pegylated interferon- in acute hepatitis C. Journal of Viral Hepatitis, 2007; 14:116-21.

10. Wiegand J., Jäckel E., Cornberg M., et al. Long-term follow-up after successful interferon therapy of acute hepatitis C. Hepatology 2004;40:98-107.

11. McKiernan S.M., Hagan R., Curry M., et al. Distinct MHC class I and II alleles are associated with hepatitis $\mathrm{C}$ viral clearance, originating from a single source. Hepatology 2004;108-14.

12. Santantonio T., Medda E., Ferrari C., et al. Risk factors and outcome among a large patient cohort with community-acquired acute hepatitis C in Italy. Clin Infect Dis 2006;43:1154-9.

13. Afdhal N.H. The natural history of hepatitis C. Seminars in Liver Diseases 2004;24,suppl. 2:3-8.

14. Strader D.B., Wright T., Thomas D.L., Seeff L.B. AASLD Practice Guideline - Diagnosis, Management and Treatment of Hepatitis C. Hepatology 2004;39:1147-71.

15. Heller T., Rehermann B. Acute hepatitis C: a multifaceted disease. Seminars in Liver Diseases 2005;25:7-17.

16. Alberti A., Boccato S., Vario A., Benvegnù L. Therapy of acute hepatitis. Hepatology 2002;5:S195-S200.

17. Micallef J.M., Kaldor J.M., Dore G.J. Spontaneous viral clearance following acute hepatitis $\mathrm{C}$ infection: a systematic review of longitudinal studies. Journal of Viral Hepatitis 2006;13:34-41.

18. Nomura H., Sou S., Tanimoto H., et al. Short-term interferon-alfa therapy for acute hepatitis $\mathrm{C}$ : a randomized controlled trial. Hepatology 2004;39:1213-19.

19. Consensus Statements on the Prevention and Management of Hepatitis B and Hepatitis C in the Asia-Pacific Region. Journal of Gastroenterology and Hepatology 2000;15:815-41.

20. Kamal S.M., Fouly A.E., Kamel R.R., et al. Peginterferon alfa-2b therapy in acute hepatitis $C$ : impact of onset of therapy on sustained virologic response. Gastroenterology 2006;130:632-8.

21. De Rosa F.G., Bargiacchi O., Audagnotto S., et al. Twelve-week treatment of acute hepatitis $\mathrm{C}$ virus with pegylated interferon$\alpha-2 b$ in injection drug users. Clinical Infectious Diseases 2007; 45:583-8.

22. Alter M.J. Prevention of spread of hepatitis C. Hepatology 2002;36:suppl.1:593-8.

23. Mondelli M.U., Cerino A., Cividini A. Acute hepatitis C: diagnosis and management. Journal of Hepatology 2005;42:S108-S14.

24. Corey K.E., Ross A.S., Wurcel A., et al. Outcomes and treatment of acute hepatitis $\mathrm{C}$ virus infection in a United States population. Clinical Gastroenterology and Hepatology 2006;4:1278-82. 
25. Manns M.P., Mc Hutchison J.G., Gordon S.C., et al. Peginterferon alfa- $2 b$ plus ribavirin compared with interferon alfa- $2 b$ plus ribavirin for initial treatment of chronic hepatitis $\mathrm{C}$ : a randomized trial. Lancet 2001;358:958-65.

26. Fried M.W., Shiffman M.L., Reddy K.R., et al. Peginterferon alfa2a plus ribavirin for chronic hepatitis $\mathrm{C}$ virus infection. New England Journal of Medicine 2002;347:975-82.

27. Hofer H., Watkins-Riedel T., Janata O., et al. Spontaneous viral clearance in patients with acute hepatitis $\mathrm{C}$ can be predicted by repeated measurements of serum viral load. Hepatology 2003; $37: 60-4$.

28. Gerlach J.T., Diepolder H.M., Zachoval R., et al. Acute hepatitis C: high rate of both spontaneous and treatment-induced viral clearance. Gastroenterology 2003;125:80-8.

29. Davis G.I., Wong J.B., McHutchinson J.G., et al. Early virologic response to treatment with peginterferon alfa-2b plus ribavirin in patients with chronic hepatitis C. Hepatology 2003;38:645-52.

30. Firenci P. Predicting the therapeutic response in patients with chronic hepatitis C: the role of viral kinetics studies. J Antimicrob Chemother 2004;53:15-8.
31. Yamaji K., Hayashi J., Kawakami Y., et al. Hepatitis C viral RNA status at two weeks of therapy predicts the eventual response. J Clin Gastroenterol 1998;26:193-9.

32. Wiegand J., Baggish P., Boecher W., et al. Early monotherapy with pegylated interferon alpha- $2 b$ for acute hepatitis infection: the HEP-NET acute-HCV II study. Hepatology 2006;43:250-6.

33. Jaeckel E., Cornberg M., Waldemeyer H., et al. Acute hepatitis C: to treat or not to treat? Hepatology 2002;35:1538-40.

34. Management of hepatitis C. NIH Consensus Development Conference, 2002.

35. Angerami R.N., Stucchi R., Gonçales N.S.L., Gonçales Jr. F.L.G. Hepatite C aguda. II Consenso da Sociedade Paulista de Infectologia para Manuseio e Terapia da Hepatite C 2004:34-6.

36. Zekry A., Patel K., Mc Hutchison J.G. Treatment of acute hepatitis $\mathrm{C}$ infection: more pieces of the puzzle. Journal of Hepatology 2005;42:293-6.

37. Focaccia R., Galante V.C., Oliveira U.B. Hepatite C Epidemiologia. In: Tratado de Hepatites Virais. Ed Roberto Focaccia, $2^{\mathrm{a}}$ Edição, Editora Atheneu, 2007:211-16.

38. Consenso da Sociedade Paulista de Infectologia para Manuseio e Terapia da Hepatite C, 2004. 